\title{
Análisis de tasas y modelos para generación de viajes en hipermercados y supermercados
}

[Analysis of trip generation rates and models for hypermarkets and supermarkets]

\author{
Marcelo Herz*, Jorge Galarraga \\ Córdoba National University - Argentina, Córdoba National University - Argentina
}

Submitted 6 May 2013; received in revised form 30 Sep 2013; accepted 1 Nov 2013

\begin{abstract}
Resumen
La localización de polos generadores de viajes comerciales produce en el área de influencia impactos urbanos por aumentos de viajes motorizados que afectan la calidad de vida y el ambiente. Para mejor comprensión de este fenómeno, se reporta en el presente artículo un análisis de tasas y modelos de generación de viajes en hipermercados y supermercados efectuado con relación a variaciones en el tiempo y al porte de los establecimientos tomando experiencias de Estados Unidos. Se analizan diferentes ediciones del Trip Generation Manual publicado por el ITE (Institute of Transportation Engineers) observando que las tasas reportadas de generación de viajes motorizados disminuyen con el aumento de Área Total Construida (ATC), como variable independiente. Este efecto también se observa en modelos de Shopping Centers que abarcan los rangos de ATC de hipermercados y supermercados, lo que permite vincular estos usos del suelo. Finalmente, la relación entre generación de viajes y porte del establecimiento se prueba para casos de hipermercados en Argentina, y se formulan modelos de generación con funciones de potencia de exponente 2/3 para la variable independiente ATC.
\end{abstract}

Palabras clave: polos generadores de viajes, hipermercados, supermercados, tasas, modelos.

\begin{abstract}
The location of commercial trip generation hubs produces urban impacts in the area of influence since they increase the number of vehicle trips that affects environment and quality of life. For better understanding of this phenomenon, it is reported in this article an analysis of trip generation rates and models in hypermarkets and supermarkets considering different periods of time and size of the establishments, taking into account USA experiences. After analyzing several editions of the Trip Generation Manual published by ITE (Institute of Transportation Engineers) it is noted that the reported generation rates of vehicle trips decrease with increasing Gross Floor Area (GFA), as the independent variable. This effect is also observed in Shopping Centers models, where the ranges of GFA include those of hypermarkets and supermarkets, allowing to relate these land uses. Finally , the relationship between trip generation and size of the establishment is checked for hypermarkets in Argentina, and generation models of power functions with exponent $2 / 3$ for the independent variable GFA are proposed.
\end{abstract}

Key words: trip generation hubs, hypermarkets, supermarkets, rates, models.

*Email: mherz@efn.uncor.edu.

\section{Recommended Citation}

Herz, M. and Galarraga, J. (2014) Análisis de tasas y modelos para generación de viajes en hipermercados y supermercados. Journal of Transport Literature, vol. 8, n. 3, pp. 172-198.

- JTL/RELIT is a fully electronic, peer-reviewed, open access, international journal focused on emerging transport markets and published by BPTS - Brazilian Transport Planning Society. Website www.transport-literature.org. ISSN 2238-1031.

This paper is downloadable at www.transport-literature.org/open-access. 


\section{Introducción}

Los Polos Generadores de Viajes (PGV) se han convertido en objeto de estudio por sus impactos en la accesibilidad, movilidad, calidad de vida y ambiente de nuestras ciudades. Considerando la frecuencia de los viajes con motivo de compras, se destacan los relacionados con el abastecimiento de alimentos y artículos del hogar, que van desde almacenes de barrio hasta hipermercados. A mayor porte del comercio, mayor atracción de viajes, y con el aumento de la distancia, mayor atracción de viajes motorizados. En tanto la localización de grandes supermercados e hipermercados es propuesta por motivaciones comerciales, la gestión de las ciudades sobre los usos del suelo requiere herramientas de diagnóstico y evaluación para toma de decisiones, que incluyen una mejor comprensión de los patrones de generación de viajes asociados con las características del emprendimiento y con el contexto socioeconómico y cultural local.

El propósito del presente trabajo es avanzar en la comprensión de variables y tendencias en tasas y modelos de generación de viajes en hipermercados y supermercados. Para ello se focalizan casos de Estados Unidos de Norte América reportados en diferentes ediciones del manual Trip Generation del ITE (Institute of Transportation Engineers), evaluando la evolución en el tiempo de tasas y modelos correspondientes. Por otra parte se evalúa la variación de tasas con el porte de hipermercados y supermercados expresado en unidades de Área Total Construida (ATC) como variable independiente, y se compara con la variación de tasas de Shopping Centers, que abarcan el rango de portes de estos usos del suelo. Las comparaciones pretenden evaluar la influencia del tipo de usos de suelo comercial y del porte en el patrón de generación de viajes. Con las conclusiones de los casos reportados por el ITE, se analizan casos de hipermercados en Argentina, con la debida consideración de las diferencias de contexto.

La Sección 1 presenta antecedentes bibliográficos y caracterización de los usos del suelo considerados. En la Sección 2 se analizan tasas y modelos de generación de viajes reportados por el ITE para supermercados e hipermercados en tres usos del suelo y en nueve dimensiones temporales, para el periodo de los últimos 20 años. La Sección 3, también con datos del ITE, presenta la evaluación de relaciones en la generación de viajes entre supermercados, hipermercados y Shopping Centers y se detectan similitudes que son planteadas a nivel de modelos generalizados. En la sección 4 se describen antecedentes de estudios en Argentina y se discuten posibles aplicaciones de las caracterizaciones encontradas en los estudios ITE para 
hipermercados y supermercados locales. Finalmente se resumen las correspondientes conclusiones.

\section{Generación de viajes en Polos Comerciales}

Los Polos Generadores de Viajes (PGV) son emprendimientos de gran porte caracterizables para distintos usos del suelo con capacidad de generar grandes flujos de viajes. Originalmente fueron estudiados los impactos asociados con circulación y estacionamientos de automóviles, pero el concepto fue evolucionando para contemplar otros modos como viajes no motorizados, transporte público, y dimensiones ambientales de sustentabilidad y calidad de vida. (Portugal L.S.,2012).

El ITE comenzó a partir de la década de 1970 un programa de sistematización de datos de generación de viajes vehiculares para diversos usos del suelo y rangos de diversos tamaños en los Estados Unidos de América. En la novena edición del manual Trip Generation se contemplan 42 tipos de actividades para el uso del suelo "comercio" (ITE, 2012), de los cuales 10 se relacionan con hipermercados y supermercados, según se muestran en la Tabla 1.

Los usos del suelo más representativos de hipermercados y supermercados son el 813, 854 y 850. El uso del suelo 813 es específicamente de Hipermercados. Según ITE un Hipermercado (Free Standing Discount Superstore) se caracteriza por ofrecer una completa gama de alimentos y un área de mercaderías generales con precios rebajados, bajo el mismo techo, compartiendo entradas y salidas y con playas de estacionamiento propio. La denominación "free standing" excluye el caso de hipermercados que forman parte de Shopping Centers, tratados como uso del suelo 820. Una importante contribución con el estudio de 32 sitios de la mayor cadena de hipermercados de EEUU se debe a Pearson et al. (2009).

El uso del suelo 854 representa un Supermercado con Descuentos (Discount Supermarket) que se caracteriza por ofrecer una completa gama de alimentos y productos de limpieza y servicios del hogar con precios rebajados. El ITE describe este uso del suelo como relacionado con Supermercado (Código 850) siendo la principal diferencia el tipo de comercialización a precios con descuentos. Se interpreta que estos supermercados 
corresponden a cadenas que publicitan ofertas por medios masivos de comunicación, con establecimientos de distintos portes distribuidos geográficamente en una región.

Tabla 1 - Clasificación ITE de comercios relacionables con hipermercados y supermercados (GFA en metros cuadrados)

\begin{tabular}{|c|c|c|c|c|}
\hline $\begin{array}{l}\text { Código } \\
\text { ITE }\end{array}$ & Denominación & Rango GFA & $\begin{array}{l}\text { GFA } \\
\text { medio }\end{array}$ & Caracterización \\
\hline 813 & $\begin{array}{c}\text { Free Standing Discount } \\
\text { Superstore }\end{array}$ & $12000-23000$ & 20000 & $\begin{array}{l}\text { Hipermercado con completa } \\
\text { gama de alimentos y } \\
\text { productos de tiendas de } \\
\text { departamento, gran porte }\end{array}$ \\
\hline 814 & Specialty Retail Center & $1000-15000$ & 5000 & $\begin{array}{l}\text { Pequeños Shopping } \\
\text { Center sin alimentos }\end{array}$ \\
\hline 815 & $\begin{array}{c}\text { Free Standing Discount } \\
\text { Store }\end{array}$ & $5000-25000$ & 10000 & $\begin{array}{l}\text { Hipermercado sin } \\
\text { completa gama de } \\
\text { alimentos }\end{array}$ \\
\hline 820 & Shopping Center & $10000-160000$ & 45000 & $\begin{array}{l}\text { Gran porte. Grupo } \\
\text { integrado como unidad }\end{array}$ \\
\hline 850 & Supermarket & $2000-12000$ & 3000 & $\begin{array}{l}\text { Supermercado con } \\
\text { completa gama de } \\
\text { alimentos y otros }\end{array}$ \\
\hline $851 / 852$ & $\begin{array}{c}\text { Convenience Market (24 } \\
\text { hs/16 hs) }\end{array}$ & $100-400$ & 200 & $\begin{array}{c}\text { Pequeño porte. } \\
\text { (alimentos, revistas) }\end{array}$ \\
\hline 854 & Discount supermarket & $1000-13000$ & 8000 & $\begin{array}{l}\text { Supermercado con } \\
\text { precios rebajados } \\
\text { (cadenas regionales) }\end{array}$ \\
\hline 862 & $\begin{array}{l}\text { Home Improvement } \\
\text { Superstore }\end{array}$ & $5000-17000$ & 10000 & $\begin{array}{c}\text { Herramientas, Pinturas, } \\
\text { maderas }\end{array}$ \\
\hline 863 & Electronics Superstore & $3000-4500$ & 3500 & TV, Audio, Computación \\
\hline 869 & $\begin{array}{c}\text { Discount home furnishing } \\
\text { Superstore }\end{array}$ & $10000-50000$ & 30000 & $\begin{array}{c}\text { Gran porte. Equipamiento } \\
\text { del hogar }\end{array}$ \\
\hline
\end{tabular}

Una importante contribución con el estudio de 10 sitios de una gran cadena regional de supermercados del oeste de EEUU se debe a Brehmer y Butorac (2003). Los autores reportan estudios de generación de viajes realizados en comercios que operan 24 horas por día los 7 días de la semana, con Áreas Totales Construidas entre 7000 y $9000 \mathrm{~m}^{2}$ y que ofrecen los mismos tipos y marcas de productos que los supermercados convencionales pero a menos precio que cualquier competidor en el área. Con estas características se asocian con el uso del suelo 854 del ITE (Discount Supermarket). 
La variable independiente más utilizada para obtener las tasas y modelos de generación de viajes de los 3 usos del suelo correspondientes a hipermercados y supermercados es el Área Total Construida (GFA: Gross Floor Area) cuantificada como la suma en pies cuadrados, de las áreas de cada piso del edificio que se encuentre dentro de las caras externas de las paredes exteriores. Los viajes generados cuentan como la suma de viajes atraídos y producidos por el emprendimiento, y se reportan en varias dimensiones temporales (días y horas pico).

Los manuales del ITE se han utilizado como referencia para tasas y modelos de generación de viajes vehiculares en países latinoamericanos, no obstante las reconocidas diferencias socioeconómicas y culturales. Para disponer de referencias locales y no solo limitadas a generación de viajes vehiculares, la Red Iberoamericana de Estudio en Polos Generadores de Viajes ha reportado estudios regionales para diversos usos del suelo. (Portugal L.S., 2012).

En el caso de hipermercados y supermercados los estudios locales de generación de viajes son escasos en comparación con el ITE (Galarraga et al 2011), pudiendo citar entre los más recientes Silva y Silva (2006), Galarraga et al (2007), Freitas y Raia Jr. (2008) Herz y Galarraga (2013).

\section{Análisis de tasas y modelos ITE para hipermercados y supermercados}

El presente apartado analiza los usos del suelo ITE correspondientes a Supermercados (850), Supermercados de Cadenas Regionales (854) e Hipermercados (813) en las publicaciones del manual Trip Generation correspondientes a la Quinta Edición (ITE, 1991), Octava Edición (ITE, 2008) y Novena Edición (ITE, 2012). La selección de las ediciones responde al propósito de evaluar un periodo de largo plazo (1991-2012) y un periodo reciente de corto plazo (2008-2012). 


\subsection{Variables independientes y períodos de tiempo considerados}

En lo referente a variables independientes queda claro que el Área Total Construida (Gross Floor Área, GFA) en miles de pies cuadrados $\left(\mathrm{Kp}^{2}\right)$ ha sido seleccionada en todos los usos del suelo y ediciones analizadas.

Para el uso del suelo 813 GFA es la única variable independiente considerada; para el uso del suelo 850 en la edición 1991 se incluye además un estudio con variable independiente cantidad de empleados y para el uso del suelo 854 en la edición 2012 se incluyen además cuatro estudios con variable independiente cantidad empleados y con variable independiente volumen horario de la calle adyacente. Sobre la base de lo expuesto en el resto del documento se trabajará solamente con GFA como variable independiente para los tres tipos de uso del suelo en análisis.

En lo referente a períodos de tiempo, típicamente son nueve los considerados: a) Día de semana, b) Hora pico de la calle adyacente por la mañana (HP CA AM), c) Hora pico de la calle adyacente por la tarde (HP CA PM), d) Hora pico del generador por la mañana (HP G AM), e) Hora pico del generador por la tarde (HP G PM), f) Día Sábado, g) Hora pico del generador para el sábado (HP G S), h) Día Domingo e i) Hora pico del generador para el domingo (HP G D).

La Tabla 2 muestra, para cada período de tiempo y uso del suelo, la cantidad de estudios (C.E.) y el valor medio (V.M.) de la variable independiente (GFA en $\mathrm{Kp}^{2}$ ) en las tres ediciones en consideración. Puede advertirse que el valor medio del GFA para uso del suelo 850 se encuentra aproximadamente entre 30 y $70 \mathrm{Kp}^{2}$, para uso del suelo 854 entre 80 y 130 $\mathrm{Kp}^{2}$ y para uso del suelo 813 entre 190 y $200 \mathrm{Kp}^{2}$.

El uso del suelo 813 presenta el mayor número de estudios en casi todos los períodos horarios (entre 42 y 86 estudios), a pesar que este uso del suelo no existía en la edición de 1991, lo que muestra la creciente importancia de los hipermercados como Polos Generadores de Viajes.

El uso del suelo 850 presenta solamente dos estudios para día sábado y domingo y ambos ya estaban en la edición de 1991; la mayor cantidad de estudios se registra en los períodos pico de la calle adyacente por la tarde (62). Para el uso del suelo 854 la cantidad de estudios resulta relativamente reducida (entre 5 y 19 ). 
Tabla 2 - Cantidad de Estudios (C.E.) y Valor Medio (V.M.) variable independiente GFA(Kp $\left.{ }^{2}\right)$

\begin{tabular}{c|c|c|c|c|c|c|c}
\hline \multirow{2}{*}{ ITE Año } & Período & \multicolumn{2}{|c|}{ Uso del Suelo 850 } & \multicolumn{2}{c|}{ Uso del Suelo 854 } & \multicolumn{2}{c}{ Uso del Suelo 813 } \\
\cline { 3 - 8 } & Tiempo & C.E. & V.M. & C.E. & V.M. & C.E. & V.M. \\
\hline 1991 & Día Semana & & & & & & \\
\hline 2008 & Día Semana & 4 & 39 & 7 & 77 & 45 & 195 \\
\hline 2012 & Día Semana & 4 & 39 & 11 & 80 & 65 & 196 \\
\hline 1991 & HP CA AM & 6 & 39 & 3 & 106 & & \\
\hline 2008 & HP CA AM & 5 & 30 & 13 & 84 & 47 & 195 \\
\hline 2012 & HP CA AM & 13 & 37 & 17 & 84 & 67 & 196 \\
\hline 1991 & HP CA PM & 10 & 32 & 3 & 106 & & \\
\hline 2008 & HP CA PM & 40 & 59 & 15 & 74 & 64 & 201 \\
\hline 2012 & HP CA PM & 62 & 56 & 19 & 77 & 86 & 200 \\
\hline 1991 & HP G AM & 3 & 42 & 3 & 106 & & \\
\hline 2008 & HP G AM & 4 & 39 & 3 & 106 & 45 & 195 \\
\hline 2012 & HP G AM & 12 & 39 & 7 & 94 & 65 & 195 \\
\hline 1991 & HP G PM & 7 & 35 & 3 & 106 & & \\
\hline 2008 & HP G PM & 7 & 37 & 3 & 106 & 47 & 195 \\
\hline 2012 & HP G PM & 13 & 45 & 7 & 94 & 67 & 196 \\
\hline 1991 & Sábado & 2 & 27 & 1 & 127 & & \\
\hline 2008 & Sábado & 2 & 27 & 11 & 82 & 43 & 195 \\
\hline 2012 & Sábado & 2 & 27 & 15 & 83 & 43 & 195 \\
\hline 1991 & HP G S & 5 & 27 & 2 & 95 & & \\
\hline 2008 & HP G S & 32 & 67 & 12 & 80 & 52 & 198 \\
\hline 2012 & HP G S & 34 & 67 & 16 & 81 & 52 & 198 \\
\hline 1991 & Domingo & 2 & 27 & 1 & 127 & & \\
\hline 2008 & Domingo & 2 & 27 & 9 & 84 & 42 & 195 \\
\hline 2012 & Domingo & 2 & 27 & 13 & 84 & 42 & 195 \\
\hline 1991 & HP G D & 2 & 27 & 1 & 127 & & \\
\hline 2008 & HP G D & 2 & 27 & 1 & 127 & 42 & 195 \\
\hline 2012 & HP G D & 2 & 27 & 5 & 93 & 42 & 195 \\
\hline & & & & & & & \\
\hline
\end{tabular}

\subsection{Tasas y modelos}

La Tabla 3 muestra, para cada período de tiempo y uso del suelo, la tasa media (T.M.) para la variable independiente GFA en $\mathrm{Kp}^{2}$ y la desviación estándar (D.E.) de las tasas, en las tres ediciones en consideración. Los espacios en blanco en Tasas Medias de la Tabla corresponden a períodos de tiempo no reportados, como por ejemplo el ya citado del Uso del Suelo 813 en la edición de 1991. Para la Desviación Estándar los valores no se reportan cuando la cantidad de estudios disponibles (ver Tabla 2) son uno o dos. 
Tabla 3-Tasa Media (T.M.) y Desv. Estándar (D.E.) variable independiente GFA (Kp²) (adaptada del ITE)

\begin{tabular}{c|c|c|c|c|c|c|c}
\hline \multirow{2}{*}{ ITE Año } & \multirow{2}{*}{$\begin{array}{c}\text { Período } \\
\text { Tiempo }\end{array}$} & \multicolumn{2}{|c|}{ Uso del Suelo 850 } & \multicolumn{2}{c|}{ Uso del Suelo 854 } & \multicolumn{2}{c}{ Uso del Suelo 813 } \\
\cline { 5 - 8 } & T.M. & D.E. & T.M. & D.E. & T.M. & D.E. \\
\hline 1991 & Día Semana & & & & & & \\
\hline 2008 & Día Semana & 102,24 & 31,73 & 96,82 & 20,06 & 53,13 & 13,31 \\
\hline 2012 & Día Semana & 102,24 & 31,73 & 90,86 & 19,14 & 50,75 & 14,73 \\
\hline 1991 & HP CA AM & 2,01 & 3,05 & 1,72 & 1,43 & & \\
\hline 2008 & HP CA AM & 3,59 & 3,18 & 2,74 & 1,91 & 1,67 & 1,45 \\
\hline 2012 & HP CA AM & 3,4 & 2,64 & 2,53 & 1,84 & 1,85 & 1,55 \\
\hline 1991 & HP CA PM & 10,34 & 8,63 & 9,76 & 3,3 & & \\
\hline 2008 & HP CA PM & 10,5 & 4,97 & 8,9 & 3,32 & 4,61 & 2,37 \\
\hline 2012 & HP CA PM & 9,48 & 4,81 & 8,34 & 3,33 & 4,35 & 2,36 \\
\hline 1991 & HP G AM & 11,06 & 3,6 & 7,32 & 2,76 & & \\
\hline 2008 & HP G AM & 10,05 & 3,96 & 7,32 & 2,76 & 3,45 & 2,06 \\
\hline 2012 & HP G AM & 7,07 & 4,18 & 6,33 & 2,74 & 3,08 & 2,03 \\
\hline 1991 & HP G PM & 12,39 & 4,46 & 9,84 & 3,32 & & \\
\hline 2008 & HP G PM & 11,85 & 4,72 & 9,84 & 3,32 & 4,68 & 2,39 \\
\hline 2012 & HP G PM & 8,37 & 4,8 & 8,13 & 3,41 & 4,4 & 2,37 \\
\hline 1991 & Sábado & 177,59 & & 91,41 & & & \\
\hline 2008 & Sábado & 177,59 & & 117,03 & 22,04 & 64,07 & 16,71 \\
\hline 2012 & Sábado & 177,59 & & 111,85 & 21,63 & 64,07 & 16,71 \\
\hline 1991 & HP G S & 15,33 & 6,3 & 10,13 & & & \\
\hline 2008 & HP G S & 10,85 & 4,93 & 10,46 & 3,47 & 5,64 & 2,61 \\
\hline 2012 & HP G S & 10,65 & 4,88 & 9,65 & 3,57 & 5,64 & 2,61 \\
\hline 1991 & Domingo & 166,44 & & 65,39 & & & \\
\hline 2008 & Domingo & 166,44 & & 102,54 & 22,14 & 56,12 & 16,49 \\
\hline 2012 & Domingo & 166,44 & & 99,75 & 19,78 & 56,12 & 16,49 \\
\hline 1991 & HP G D & 18,93 & & 8,7 & & & \\
\hline 2008 & HP G D & 18,93 & & 8,7 & & 5,18 & 2,53 \\
\hline 2012 & HP G D & 18,93 & & 7,85 & 2,84 & 5,18 & 2,53 \\
\hline & & & & & & & \\
\hline
\end{tabular}

Puede advertirse como las Tasas Medias por GFA son mayores para el Uso del Suelo 850, algo menores para el suelo 854 y mucho menores para el Uso de Suelo 813. Es decir, las tasas disminuyen a medida que aumenta el Área Total Construida. 
La hora pico de la calle adyacente por la tarde presenta tasas muy superiores (del orden del triple o doble según los casos) que la hora pico de la calle adyacente por la mañana. Las tasas de días sábado son las más altas, del orden del 25\% más que las de un día de semana y del $12 \%$ más que las de los días domingo. Las tasas correspondientes a la hora pico del generador se encuentran en el orden del 8 al 10\% de las tasas diarias.

Comparando las tasas de la edición ITE 2012 con las correspondientes al ITE 2008 puede advertirse como consistentemente las del 2012 resultan menores. Los porcentajes de reducción son variables, pero en términos generales resultan del 5 al 30\% para el Uso del Suelo 850, del 5 al 15\% para el Uso del Suelo 854 y del 5 al 10\% para el Uso del Suelo 813.

Teniendo en cuenta que las ediciones del ITE calculan las tasas medias considerando todos los estudios disponibles, los nuevos estudios incorporados entre 2008 y 2012 deben presentar tasas medias aún menores a las reportadas en la última edición.

La Tabla 4 ofrece los modelos disponibles para los diferentes períodos de tiempo y usos del suelo. Corresponde destacar que el ITE solamente presenta modelos en los casos que el coeficiente de determinación $\mathrm{R}^{2}$ sea mayor a 0,50; que la variable dependiente (viajes generados en automóviles) aumente con un incremento de la variable independiente y que la cantidad de estudios sea mayor o igual a cuatro. De la Tabla 4 puede determinarse, para el caso del Uso del Suelo 813, que en el año 2012 la cantidad de estudios en algunos períodos de tiempo es mayor y en otros igual a la cantidad de estudios incluidos en la edición 2008. Sin embargo los modelos son únicamente reportados en el 2008 y no nuevamente en el 2012.

Puede advertirse como algunos de los modelos para los Usos del Suelo 850 y 854 presentan funciones lineales y otros presentan funciones de potencia linealizadas con logaritmos. En cambio para el uso del Suelo 813 todos los modelos son funciones de potencia linealizadas con logaritmos. 
Tabla 4 - Modelos para variable independiente GFA en $\mathbf{K p}^{\mathbf{2}}$ ( adaptada del ITE)

\begin{tabular}{|c|c|c|c|c|}
\hline $\begin{array}{l}\text { ITE } \\
\text { Año }\end{array}$ & $\begin{array}{l}\text { Período } \\
\text { Tiempo }\end{array}$ & Uso Suelo 850 & Uso Suelo 854 & Uso Suelo 813 \\
\hline 2008 & $\begin{array}{c}\text { Día } \\
\text { Semana }\end{array}$ & $\begin{array}{c}\mathrm{Y}=66,95(\mathrm{X})+ \\
1391,56 \quad\left(\mathrm{R}^{2}=0,52\right)\end{array}$ & & $\begin{array}{c}\operatorname{Ln}(Y)=1,35 \operatorname{Ln}(X)+ \\
2,11 \quad\left(R^{2}=0,54\right)\end{array}$ \\
\hline 2012 & $\begin{array}{c}\text { Día } \\
\text { Semana }\end{array}$ & $\begin{array}{c}Y=66,95(X)+ \\
1391,56 \quad\left(R^{2}=0,52\right)\end{array}$ & & \\
\hline 2008 & $\begin{array}{c}\text { HP CA } \\
\text { PM }\end{array}$ & $\begin{array}{c}\operatorname{Ln}(Y)=0,61 \operatorname{Ln}(X)+ \\
3,95 \quad\left(R^{2}=0,52\right)\end{array}$ & $\begin{array}{c}\operatorname{Ln}(Y)=0,90 \operatorname{Ln}(X)+ \\
2,59 \quad\left(\mathrm{R}^{2}=0,93\right)\end{array}$ & \\
\hline 2012 & $\begin{array}{c}\text { HP CA } \\
\text { PM }\end{array}$ & $\begin{array}{c}\operatorname{Ln}(Y)=0,74 \operatorname{Ln}(X)+ \\
3,25 \quad\left(\mathrm{R}^{2}=0,52\right)\end{array}$ & $\begin{array}{c}\operatorname{Ln}(\mathrm{Y})=0,87 \mathrm{Ln}(\mathrm{X})+ \\
2,69 \quad\left(\mathrm{R}^{2}=0,89\right)\end{array}$ & \\
\hline 2008 & $\begin{array}{c}\mathrm{HPG} \\
\mathrm{AM}\end{array}$ & $\begin{array}{c}\mathrm{Y}=12,87(\mathrm{X})-109,76 \\
\left(\mathrm{R}^{2}=0,91\right)\end{array}$ & & \\
\hline 2012 & $\begin{array}{c}\mathrm{HPG} \\
\mathrm{AM}\end{array}$ & & $\begin{array}{c}\mathrm{Y}=8,06(\mathrm{X})-162,89 \\
\left(\mathrm{R}^{2}=0,79\right)\end{array}$ & \\
\hline 1991 & $\begin{array}{c}\mathrm{HP} \mathrm{G} \\
\mathrm{PM}\end{array}$ & $\begin{array}{l}\operatorname{LnY}=0,952 \operatorname{Ln}(\mathrm{X}) \\
+2,679\left(\mathrm{R}^{2}=0,85\right)\end{array}$ & & \\
\hline 2008 & $\begin{array}{c}\mathrm{HP} \mathrm{G} \\
\mathrm{PM}\end{array}$ & $\begin{array}{c}\mathrm{Y}=8,84(\mathrm{X})+111,54 \\
\left(\mathrm{R}^{2}=0,61\right)\end{array}$ & & $\begin{array}{l}\operatorname{Ln}(Y)=1,32 \operatorname{Ln}(\mathrm{X})- \\
0,16 \quad\left(\mathrm{R}^{2}=0,52\right)\end{array}$ \\
\hline 2012 & $\begin{array}{c}\text { HP G } \\
\text { PM }\end{array}$ & & $\begin{array}{c}\mathrm{Y}=10,87(\mathrm{X})-256,77 \\
\left(\mathrm{R}^{2}=0,70\right)\end{array}$ & \\
\hline 2008 & Sábado & & & $\begin{array}{c}\mathrm{LnY}=1,45 \operatorname{Ln}(\mathrm{X})+ \\
1,74 \quad\left(\mathrm{R}^{2}=0,55\right)\end{array}$ \\
\hline 1991 & HP G S & $\begin{array}{c}\operatorname{Ln}(Y)=2,065 \operatorname{Ln}(X)- \\
0,896 \quad\left(R^{2}=0,88\right)\end{array}$ & & \\
\hline 2008 & HP G S & & $\begin{array}{c}\mathrm{Y}=6,91(\mathrm{X})+285,03 \\
(\mathrm{R} 2=0,56)\end{array}$ & $\begin{array}{cl}\operatorname{Ln}(Y) & =1,40 \operatorname{Ln}(X)- \\
0,43 & \left(R^{2}=0,57\right)\end{array}$ \\
\hline 2012 & HP G S & $\begin{array}{cc}\operatorname{Ln}(Y)= & 0,57 \operatorname{Ln}(X)+ \\
4,18 & \left(\mathrm{R}^{2}=0,56\right)\end{array}$ & & \\
\hline 2008 & Domingo & & & $\begin{array}{c}\operatorname{Ln}(Y)=1,74 \operatorname{Ln}(X)+ \\
0,09 \quad\left(R^{2}=0,63\right)\end{array}$ \\
\hline 2008 & HP G D & & & $\begin{array}{c}\operatorname{Ln}(Y)=1,48 \operatorname{Ln}(X)- \\
0,92 \quad\left(R^{2}=0,64\right)\end{array}$ \\
\hline
\end{tabular}




\section{Relación con generación de viajes de Shopping Centers ITE}

Las características de usos del suelo comercial han tradicionalmente clasificado por separado los supermercados/hipermercados y los centros de compras con diversos negocios agrupados. Sin embargo muchos Shopping Centers tienen supermercados o hipermercados como tiendas anclas, y puede argumentarse que las tasas de generación de viajes comparten similitudes. Esto fue demostrado en un estudio de comparación de tasas relacionadas con el Área Total Construida para distintos usos del suelo en Nueva Zelanda y en Gran Bretaña (Milne et al, 2009). Utilizando la base de datos TRICS (Trip Rate Information Computer System) de Gran Bretaña, se encontraron las tasas de generación que muestra la Tabla 5, expresadas en viajes atraídos y producidos en hora pico por $100 \mathrm{~m} 2$ Gross Floor Area .

Tabla 5 - Tasas Medias (T.M) generación de viajes en Gran Bretaña (Milne et al,2009)

\begin{tabular}{c|c|c|c|c|c|c}
\hline \multirow{2}{*}{ GFA (m2) } & \multicolumn{3}{|c|}{ Supermercados } & \multicolumn{3}{c}{ Shopping Centers } \\
\cline { 2 - 7 } & C.E. & T.M & D.E. & C.E. & T.M. & D.E. \\
\hline $0-2000$ & 7 & 19.42 & 6.55 & 7 & 21.15 & 13.61 \\
\hline $2001-4000$ & 35 & 13.96 & 5.3 & 3 & 13.78 & 1.91 \\
\hline $4001-6000$ & 33 & 12.35 & 4.14 & 5 & 11.83 & 1.68 \\
\hline $6001-10000$ & 30 & 12.43 & 2.52 & 2 & 9.12 & - \\
\hline
\end{tabular}

C.E: Cantidad de Estudios; D.E.: Desviación Estándar

Se observa que tanto en Supermercados como en Shopping Centers las tasas son decrecientes con el aumento de porte, y que los valores son suficientemente similares como para poder combinarse en una única base de datos.

Considerando que en los casos del ITE los usos del suelo 850, 854 y 813 también muestran tasas de generación de viajes decrecientes con el Área Total Construida y que el uso del suelo 820 (Shopping Centers) abarca rangos de superficies muy amplios de 100 a 2200 Kp2 y presenta una importante cantidad de estudios acumulados, se procedió a realizar comparaciones para evaluar la influencia del tipo de usos de suelo comercial y del porte en el patrón de generación de viajes. 


\subsection{Uso del suelo 820 ITE comparados con Usos del suelo 850, 854 y 813}

Los Shopping Centers (uso del suelo 820) presentan ecuaciones de generación de viajes con buenos coeficientes de correlación en varias dimensiones temporales $\left(\mathrm{R}^{2}\right.$ del orden de $\left.80 \%\right)$ utilizando el Área Total Rentable (GLA: Gross Leasable Área) como variable independiente.

Los modelos reportados de generación de viajes son linealizaciones logarítmicas de funciones de potencia con exponentes menores a uno, que reflejan disminución de tasa de generación con el aumento de porte del Polo, similar a lo observado al comparar tasas de distintas dimensiones temporales entre supermercados (850) supermercados de cadenas regionales (854) e hipermercados (813). Con este marco y los antecedentes de similitudes encontradas en las tasas de generación entre supermercados y Shopping Centers en Gran Bretaña, se planteó evaluar el poder predictivo de los modelos del uso del suelo 820 (Shopping Centers) para reproducir las tasas medias para portes medios de supermercados e hipermercados reportadas en los usos del suelo 850, 854 y 813.

En las dimensiones temporales se evaluaron cuatro casos: Día de semana completo, Hora pico de la Calle Adyacente por la tarde (HP CA PM), Día sábado completo y Hora pico del Generador en sábado (HP G S). El procedimiento consistió en utilizar los modelos de Shopping Centers correspondientes a cada dimensión temporal para estimar los viajes generados por un supermercado con Área Total Construida (GFA) igual al porte medio del uso de suelo 850. Dividiendo el número de viajes por la misma área, se obtuvo una tasa representativa del porte medio que se comparó con la reportada por el ITE. En forma análoga se estimaron tasas para los supermercados de cadenas regionales (853) y para hipermercados (813).

La variable independiente Área Total Construida (GFA) utilizada para supermercados e hipermercados fue directamente incluida en los modelos de Shopping Centers en reemplazo de la variable independiente Área Total Rentable (GLA). Esta sustitución es consistente con las definiciones que hace el ITE de GLA aplicable a Shopping Centers como el área total disponible por los tenedores, y de GFA como el área total construida aplicable a supermercados e hipermercados aislados (free standing), o sea no dentro de un Shopping Center. 
Para Día de semana completo, la edición 2012 del ITE reporta 302 estudios de Shopping Centers, con un rango de portes de 100 a $1500 \mathrm{Kp}^{2}$, y propone el modelo de la ecuación (1)

$$
\operatorname{Ln}(\mathrm{Y})=0,65 \operatorname{Ln}(\mathrm{X})+5,83 \quad(\mathrm{R} 2=0,79)
$$

Dónde Y: viajes vehiculares atraídos y producidos en día hábil completo

X: Área Total Rentable (GLA) expresada en 1000 pies cuadrados $\left(\mathrm{Kp}^{2}\right)$

La Tabla 6 muestra datos y resultados de la estimación de tasas para día hábil completo en los usos del suelo 850, 854 y 813 utilizando la ecuación (1) con X como Área Total Construida (GFA). Se observa que la cantidad de estudios disponibles para estos usos del suelo es sustancialmente menor a los 302 estudios que sustentan la regresión de la ecuación (1). En esta dimensión temporal, la edición 2012 del ITE presenta una función lineal para supermercado y ningún modelo para los usos del suelo 854 y 813, según reportado en la Tabla 4.

Valuando X en la ecuación (1) con el Área Total Construida (GFA) del porte medio del uso del suelo correspondiente, se obtienen tasas medias con diferencias entre $-19,3 \%$ y $+5,2 \%$ respecto a las reportadas para los supermercados e hipermercados.

Tabla 6 - Tasas Medias (T.M.) Día de semana

\begin{tabular}{c|c|c|c}
\hline Día de semanal, completo & Supermercado & Supermercado CR & Hipermercado \\
\hline Uso del suelo ITE & 850 & 854 & 813 \\
\hline Cantidad de estudios C.E. & 4 & 11 & 65 \\
\hline Rango de porte $\left(\mathrm{Kp}^{2}\right)$ & $20-60$ & $65-95$ & $120-250$ \\
\hline GFA medio $\left(\mathrm{Kp}^{2}\right)$ & 39 & 80 & 196 \\
\hline $\begin{array}{c}\text { T.M. (viajes generados/Kp } \\
\text { GFA) }\end{array}$ & 102 & 91 & 51 \\
\hline $\begin{array}{c}\text { Viajes estimados con ecuación } \\
(1) \quad \text { X=GFA medio }\end{array}$ & 3682 & 5874 & 10517 \\
\hline $\begin{array}{c}\text { T.M. calculada con Ecuación } \\
(1)(\text { viajes/Kp }\end{array}$ de GFA) & 94,4 & 73,4 & 53,7 \\
\hline Diferencia & $-7,4 \%$ & $-19,3 \%$ & $5,2 \%$ \\
\hline
\end{tabular}


Para Hora pico de día de semana en calle adyacente por la tarde (HP CA PM), la edición 2012 del ITE reporta 426 estudios de Shopping Centers, con un rango de portes de 100 a $2200 \mathrm{Kp}^{2}$, y propone el modelo de la ecuación (2)

$$
\operatorname{Ln}(\mathrm{Y})=0,67 \operatorname{Ln}(\mathrm{X})+3,31 \quad\left(\mathrm{R}^{2}=0,81\right)
$$

Dónde Y: viajes vehiculares atraídos y producidos en hora pico de día hábil

$$
\text { X: Área Total Rentable (GLA) expresada en } 1000 \text { pies cuadrados }\left(\mathrm{Kp}^{2}\right)
$$

La Tabla 7 muestra datos y resultados de la estimación de tasas de generación de viajes en hora pico de día hábil en calle adyacente para los usos del suelo 850, 854 y 813 utilizando la ecuación (2). Según reportado en la Tabla 3, la edición 2012 del ITE, propone funciones de potencia con modelos linealizados con logaritmos para supermercados locales y regionales y no propone modelo para hipermercados. La cantidad de estudios disponibles para formular tasas y modelos en estos usos del suelo es sustancialmente menor a los 426 estudios que sustentan la regresión de la ecuación (2). Valuando X en la ecuación (2) con el Área Total Construida (GFA) del porte medio del uso del suelo correspondiente, las tasas medias obtenidas con la ecuación arrojan diferencias entre $-23,1 \%$ y $+11,6 \%$ respecto a las reportadas para los supermercados e hipermercados.

Tabla 7 - Tasas Medias (T.M.) en Hora Pico de calle adyacente por la tarde

\begin{tabular}{c|c|c|c}
\hline $\begin{array}{c}\text { Día hábil, Hora pico de la } \\
\text { tarde calle adyacente }\end{array}$ & Supermercado & Supermercado CR & Hipermercado \\
\hline Uso del suelo ITE & 850 & 854 & 813 \\
\hline Cantidad de estudios C.E. & 62 & 19 & 86 \\
\hline Rango de porte $\left(\mathrm{Kp}^{2}\right)$ & $10-150$ & $10-130$ & $120-250$ \\
\hline GFA medio $\left(\mathrm{Kp}^{2}\right)$ & 56 & 77 & 200 \\
\hline $\begin{array}{c}\text { T.M. (viajes generados/Kp } \\
\text { GFA) }\end{array}$ & 9,5 & 8,3 & 4,3 \\
\hline $\begin{array}{c}\text { Viajes estimados con ecuación } \\
(2) \quad \mathrm{X}=\mathrm{GFA} \text { medio }\end{array}$ & 406 & 503 & 953 \\
\hline $\begin{array}{c}\text { T.M. calculada con Ecuación } \\
(2)(\text { viajes/Kp }\end{array}$ de GFA) & 7,3 & 6,5 & 4,8 \\
\hline Diferencia & $-23,1 \%$ & $-21,7 \%$ & $11,6 \%$ \\
\hline
\end{tabular}


Para Día Sábado completo, la edición 2012 del ITE reporta 123 estudios de Shopping Centers, cantidad importante pero notablemente inferior a los estudios reportados para día de semana completo y en hora pico de calle adyacente. El rango de portes de los casos estudiados es de 50 a $1500 \mathrm{Kp}^{2}$ y para esta dimensión temporal se propone el modelo de la ecuación (3)

$$
\operatorname{Ln}(\mathrm{Y})=0,63 \operatorname{Ln}(\mathrm{X})+6,23 \quad\left(\mathrm{R}^{2}=0,82\right)
$$

Dónde Y: viajes vehiculares atraídos y producidos en día sábado completo

X: Área Total Rentable (GLA) expresada en 1000 pies cuadrados $\left(\mathrm{Kp}^{2}\right)$

La Tabla 8 muestra datos y resultados de la estimación de tasas para día hábil completo en los usos del suelo 850,854 y 813 utilizando la ecuación (3).

Tabla 8 - Tasas Medias (T.M.) Día Sábado

\begin{tabular}{c|c|c|c}
\hline Día sábado, completo & Supermercado & Supermercado CR & Hipermercado \\
\hline Uso del suelo ITE & 850 & 854 & 813 \\
\hline Cantidad de estudios C.E. & 2 & 15 & 43 \\
\hline Rango de porte $\left(\mathrm{Kp}^{2}\right)$ & $20-35$ & $60-130$ & $120-230$ \\
\hline GFA medio $\left(\mathrm{Kp}^{2}\right)$ & 27 & 83 & 195 \\
\hline $\begin{array}{c}\text { T.M. (viajes generados/Kp } \\
\text { de GFA) }\end{array}$ & 178 & 112 & 64 \\
\hline $\begin{array}{c}\text { Viajes estimados con } \\
\text { ecuación }(3) \quad \mathrm{X}=\mathrm{GFA} \text { medio }\end{array}$ & 4050 & 8216 & 14073 \\
\hline $\begin{array}{c}\text { T.M. calculada con ecuación } \\
(3)(\text { viajes/Kp }\end{array}$ de GFA) & 150,0 & 99,0 & $12,8 \%$ \\
\hline Diferencia & $-15,7 \%$ & $-11,6 \%$ & \\
\hline
\end{tabular}

Ninguno de los usos del suelo de supermercados e hipermercados presenta modelos para día sábado completo en la edición 2012 del ITE. La cantidad de estudios disponibles para estos usos del suelo es sustancialmente menor a los que sustentan la regresión de la ecuación (3). Las tasas medias muestran diferencias entre $-15,7 \%$ y $+12,8 \%$ respecto a las reportadas para los supermercados e hipermercados. 
Para Hora pico del Polo en sábado, la edición 2012 del ITE reporta 128 estudios de Shopping Centers, con un rango de portes de 50 a $1500 \mathrm{Kp} 2$, y propone el modelo de la ecuación (4)

$$
\operatorname{Ln}(\mathrm{Y})=0,65 \operatorname{Ln}(\mathrm{X})+3,78 \quad\left(\mathrm{R}^{2}=0,83\right)
$$

Dónde Y: viajes vehiculares atraídos y producidos en hora pico del Polo en día sábado

$$
\text { X: Área Total Rentable (GLA) expresada en } 1000 \text { pies cuadrados }\left(\mathrm{Kp}^{2}\right)
$$

Sólo el uso del suelo 813 tiene un modelo linealizado con logaritmos propuesto para hora pico del día sábado en la edición 2012 del ITE y aquí también se observa que la cantidad de estudios disponibles para estos usos del suelo es sustancialmente menor a los 128 estudios que sustentan la regresión de la ecuación (4). La Tabla 9 muestra en forma análoga a las tablas anteriores, tasas medias obtenidas que arrojan diferencias entre $-4,7 \%$ y $+23,2 \%$ respecto a las reportadas para los supermercados e hipermercados.

\section{Tabla 9 - Tasas Medias (T.M.) en Hora Pico del Generador en Sábado}

\begin{tabular}{c|c|c|c}
\hline $\begin{array}{c}\text { Día Sábado, Hora pico del } \\
\text { Polo Generador de Viajes }\end{array}$ & Supermercado & Supermercado CR & Hipermercado \\
\hline Uso del suelo ITE & 850 & 854 & 813 \\
\hline Cantidad de estudios C.E. & 34 & 16 & 52 \\
\hline Rango de porte $\left(\mathrm{Kp}^{2}\right)$ & $15-150$ & $60-130$ & $120-230$ \\
\hline GFA medio $\left(\mathrm{Kp}^{2}\right)$ & 67 & 81 & 198 \\
\hline $\begin{array}{c}\text { T.M. (viajes generados/Kp } \\
\text { de GFA) }\end{array}$ & 10,6 & 9,6 & 5,6 \\
\hline $\begin{array}{c}\text { Viajes estimados con } \\
\text { ecuación (4) X=GFA medio }\end{array}$ & 674 & 762 & 1363 \\
\hline $\begin{array}{l}\text { T.M. calculada con ecuación } \\
\text { (4) (viajes/Kp }{ }^{2} \text { de GFA) }\end{array}$ & 10,1 & 9,4 & 6,9 \\
\hline Diferencia & $-4,7 \%$ & $-2,0 \%$ & $23,2 \%$ \\
\hline
\end{tabular}

Los resultados de las tablas 6 a 9 pueden interpretarse como confirmando que las tasas medias de generación de viajes de los Shopping Centers y de los supermercados e hipermercados están más influenciadas por el tamaño medio del Polo comercial (Variable X) que por la categoría de uso del suelo. Siendo experimentalmente notable la disminución de tasas con el aumento de porte, los modelos con funciones lineales y las tasas medias para rangos amplios de portes tendrían limitado poder predictivo frente a los modelos basados en funciones de potencia con exponente menor a 1 . 
Por otra parte los modelos disponibles para usos del suelo 850, 853 y 813 en las cuatro dimensiones temporales evaluadas de la Edición 2012 del ITE cubren sólo 5 de los 12 casos, según reportados en la ya citada Tabla 4, uno con función lineal y cuatro con funciones de potencia, con exponentes que varían de 0,57 a 1,45; obtenidos con regresiones de datos entre 4 y 62 estudios. Según se ha mostrado en las Tablas 6 a 9, las mismas ecuaciones (1) a (4) de Shopping Centers pueden razonablemente pronosticar los viajes generados tanto en supermercados locales y regionales como en hipermercados, en las cuatro dimensiones temporales.

\subsection{Posibilidad de plantear un modelo generalizado para supermercados, hipermercados y}

\section{Shopping Centers}

Los modelos de las ecuaciones (1) a (4) para días viernes y sábado, completo y horas pico, presentan una notable similitud en los exponentes de las funciones de potencia entre 0,63 y 0,67. Las funciones de potencia son interesantes por sus propiedades de invariancia de escala (multiplicar el argumento $\mathrm{X}$ con un factor constante c, escala la función sin modificar la relación) y de universalidad (refleja fenómenos que comparten las mismas dinámicas).

Si bien cada modelo linealizado en logaritmos presenta el coeficiente estadísticamente mejor ajustado a los estudios específicos, el objetivo de los modelos es capturar lo esencial de los fenómenos observados, en este caso la generación de viajes de Shopping Centers, y en supermercados e hipermercados en distintas dimensiones temporales. Estableciendo preferencia por la simplicidad, puede aplicarse el criterio de la navaja de Ockham aceptando que en igualdad de condiciones la explicación más simple y suficiente es la más probable (Baker, 2004)

El caso con más estudios (426) corresponde a la ecuación (2), que para la hora pico por la tarde en día de semana en calle adyacente presenta un coeficiente de 0,67, algebraicamente 2/3, por lo que puede proponerse la ecuación (5) como modelo generalizado para Shopping Centers, Hipermercados y Supermercados. 


$$
\mathrm{Y}=\alpha \mathrm{X}^{2 / 3}
$$

Y: Viajes Vehiculares atraídos y producidos

X: Área Total Rentable (GLA) en Shopping Centers, Área Total Construida (GFA) en Hipermercados y Supermercados

$\alpha$ : Parámetro del modelo, depende de las unidades de X y de la dimensión temporal

Al forzar el exponente al valor $2 / 3$ debe calcularse el $\alpha$, para ello se estimaron valores de $\mathrm{Y}$ utilizando las ecuaciones (1) a (4) con el X medio de los estudios reportados en el uso del suelo 820 expresado en $\mathrm{Kp}^{2}$, resultando los modelos generalizados que muestra la Tabla 10

Tabla 10 - Modelos generalizados para los usos del suelo 820,850, 854 y 813 (X en Kp ${ }^{2}$ )

\begin{tabular}{l|c|c|c}
\hline Dimensión Temporal & X medio 820 & $\mathrm{Y}$ & Modelo \\
\hline Día de semana completo & 331 & 14785 & $\mathrm{Y}=309 \mathrm{X}^{2 / 3}$ \\
\hline Hora Pico Calle Adyacente PM & 376 & 1455 & $\mathrm{Y}=28 \mathrm{X}^{2 / 3}$ \\
\hline Sábado completo & 450 & 23833 & $\mathrm{Y}=406 \mathrm{X}^{2 / 3}$ \\
\hline Hora Pico Generador Sábado & 458 & 2351 & $\mathrm{Y}=40 \mathrm{X}^{2 / 3}$ \\
\hline
\end{tabular}

Considerando la dispersión que exhiben los estudios reportados en los gráficos de la Edición 2012 ITE, estos modelos aproximan muy razonablemente la cantidad de viajes vehiculares de las 16 combinaciones de usos del suelo y dimensiones temporales evaluadas, siempre considerando la variable independiente en términos de Área Rentable Construida (GLA) en el caso de Shopping Centers y de Área Total Construida (GFA) en el caso de supermercados e hipermercados.

La constancia del exponente 2/3 aplicado al Área Total Construida como ley de crecimiento de viajes vehiculares generados por Shopping Centers, super e hipermercados en diferentes dimensiones temporales, implica que la cantidad de viajes generados crece menos que proporcional al porte del emprendimiento. Suponiendo que el beneficio que obtienen los consumidores por la concentración de la oferta crece directamente proporcional al porte del polo, los comercios de gran tamaño tendrían la ventaja de generar menos cantidad de viajes vehiculares en la ciudad, aunque podrían ser de mayor distancia. 


\section{Aplicaciones para hipermercados y supermercados en Argentina}

Los hipermercados ingresaron en las principales ciudades Argentinas como fenómeno de la década de 1990, produciendo cambios en la frecuencia y modos de transporte para compras de alimentos y consumos del hogar, con mayor uso de viajes en auto y con picos los fines de semana, en reemplazo de viajes diarios no motorizados en comercios cercanos.

Estudios realizados en la ciudad de Córdoba (Galarraga et al., 2007) caracterizaron 7 casos de 3 cadenas de hipermercados (Wal-Mart, Carrefour, Libertad) entre las 17 y las $20 \mathrm{hs,}$ incluyendo la hora pico del días viernes en calles adyacentes y la hora pico del Polo en días sábado, para portes en el rango de 5500 a $23600 \mathrm{~m} 2$ de Área Total Construida (ATC). Realizando conteos vehiculares y encuestas en los Polos se caracterizaron distancias de los viajes, distribución modal de los viajes y tipo de viajes en auto.

En la Tabla 11 se categorizan las distancias de viaje en 6 zonas, abarcando la zona 1 un radio de hasta $1 \mathrm{~km}$ del hipermercado, y radios crecientes hasta la zona 5 que llega al límite de la ciudad, siendo la zona 6 para viajes que vienen de afuera de la ciudad. Para cada zona se reporta el porcentaje de los viajes al hipermercado y la distancia media de los viajes.

Tabla 11 - Distribución de viajes por área de origen del viaje

\begin{tabular}{c|c|c|c|c|c|c}
\hline Hipermercado & $\begin{array}{c}\text { Zona 1 } \\
<1 \mathrm{~km} \\
(\%)\end{array}$ & $\begin{array}{c}\text { Zona 2 } \\
1-2 \mathrm{~km} \\
(\%)\end{array}$ & $\begin{array}{c}\text { Zona 3 } \\
2-3 \mathrm{~km} \\
(\%)\end{array}$ & $\begin{array}{c}\text { Zona 4 } \\
(\%)\end{array}$ & $\begin{array}{c}\text { Zona 5 } \\
\text { 4km-limite } \\
\text { ciudad }(\%)\end{array}$ & $\begin{array}{c}\text { Zona 6 (fuera de la } \\
\text { ciudad) }\end{array}$ \\
\hline $\begin{array}{c}\text { Viajes promedio } \\
(\%)\end{array}$ & 18 & 23 & 15 & 9 & 23 & 12 \\
\hline $\begin{array}{c}\text { Distancia media de } \\
\text { viaje }(\mathrm{km})\end{array}$ & 0,7 & 1,7 & 2,7 & 3,7 & 8,0 & 36,0 \\
\hline
\end{tabular}

La Tabla 12 muestra la distribución modal de los viajes por zonas, siendo el porcentaje de participación de autos y ómnibus máximo para las zonas más alejadas (5 y 6), el porcentaje de participación de taxis máximo para zonas intermedias $(2,3$ y 4) y el de viajes a pie máximo para la zona 1 , de hasta $1 \mathrm{~km}$. 
Tabla 12- Distribución modal por zonas

\begin{tabular}{c|c|c|c|c|c}
\hline Zona & $\begin{array}{c}\text { Auto } \\
(\%)\end{array}$ & Taxi (\%) & Ómnibus $(\%)$ & A pie $(\%)$ & Otros $(\%)$ \\
\hline 1 & 43,9 & 5,1 & 1,4 & 46,0 & 3,6 \\
\hline 2,3 e 4 & 60,6 & 14,3 & 7,8 & 9,7 & 7,6 \\
\hline 5 e 6 & 82,9 & 3,1 & 10,9 & 0,0 & 3,1 \\
\hline Promedio & 69.6 & 6.2 & 5.5 & 13.7 & 4.9 \\
\hline
\end{tabular}

En la Tabla 13 se categorizan los tipos de viajes en auto en primarios, exclusivos con destino al hipermercado; viajes desviados, con otro destino y cambiando parcialmente el itinerario para llegar al hipermercado, y viajes pasantes, con otro destino pero pasando por el hipermercado sin modificar su ruta. Con esta categorización, que facilita evaluar las cargas de tránsito en la red, se reportan los porcentajes de cada tipo de viajes para tres grupos de zonas. Se observa que la menor cantidad de viajes primarios proviene de las zonas más alejadas.

Tabla 13 - Distribución de viajes por tipo según zonas

\begin{tabular}{c|c|c|c}
\hline Zona & Viajes primarios $(\%)$ & Viajes desviados $(\%)$ & Viajes pasantes $(\%)$ \\
\hline 1 & 92,4 & 5,7 & 1,9 \\
\hline 2,3 e 4 & 83,7 & 9,0 & 7,3 \\
\hline 5 e 6 & 70,4 & 16,9 & 12,7 \\
\hline Promedio & 82.6 & 10.1 & 7.2 \\
\hline
\end{tabular}

Con los conteos de los viajes vehiculares (autos + taxis) atraídos y producidos en las horas pico de viernes y sábado, se estimaron mediante regresión lineal modelos de generación con tres variables independientes: Área Total Construida (ATC); Área de Ventas (AV) y Líneas de cajas. La variable independiente ATC, en forma similar a la definición de Gross Floor Área del ITE, incluye las áreas de cada piso del edificio que se encuentran dentro de las caras externas de las paredes exteriores, pero siendo en este caso la superficie medida en $\mathrm{m}^{2}$. La variable independiente AV considera solo las superficies del ATC habilitadas a los clientes, excluyendo áreas de depósitos y oficinas, también medida en $\mathrm{m}^{2}$. La variable independiente Línea de cajas cuantifica la cantidad de cajas instaladas en el hipermercado para el pago de los productos.

La Tabla 14 muestra los modelos obtenidos para hora pico del viernes en calle adyacente y hora pico del hipermercado en sábado para las tres variables independientes, con sus respectivos $\mathrm{R}^{2}$. En todos los casos los estadísticos t resultaron mayores a 2,5. 
Tabla 14 - Modelos de generación de viajes vehiculares en horas pico de días viernes y sábado

\begin{tabular}{c|c|c|c|c}
\hline Variable & $\begin{array}{c}\text { Modelos hora pico calle } \\
\text { adyacente en Viernes }\end{array}$ & $\mathrm{R}^{2}$ & $\begin{array}{c}\text { Modelos hora pico } \\
\text { Generador en Sábado }\end{array}$ & $\mathrm{R}^{2}$ \\
\hline ATC $(\mathrm{m} 2)$ & $\mathrm{Ln} \mathrm{Y}=0,92 \mathrm{Ln} \mathrm{X}-2,45$ & 0,56 & $\mathrm{Ln} \mathrm{Y}=0,93 \mathrm{Ln} \mathrm{X}-2,18$ & 0,56 \\
\hline $\mathrm{AV}(\mathrm{m} 2)$ & $\mathrm{Ln} \mathrm{Y}=1,07 \mathrm{Ln} \mathrm{X}-3,00$ & 0,71 & $\mathrm{Ln} \mathrm{Y}=1,08 \operatorname{Ln} \mathrm{X}-2,75$ & 0,71 \\
\hline Líneas de cajas $\left(\mathrm{N}^{\circ}\right)$ & $\mathrm{Y}=36,14 \mathrm{X}-861,30$ & 0,85 & $\mathrm{Y}=54,51 \mathrm{X}-1322,27$ & 0,85 \\
\hline
\end{tabular}

Si bien el modelo lineal con la cantidad de cajas presenta el mejor $\mathrm{R}^{2}$, la variable más adecuada para planificación y decisiones sobre permisos de localización y/o de ampliación es el Área Total a Construir (ATC), pues tanto el Área de Ventas como el Número de líneas de caja son variables del layout interno, de la distribución de depósitos, y modificables para una misma ATC. Se observa que los modelos con variable ATC son los de peor $\mathrm{R}^{2}$, pero representan una función de potencia linealizada con logaritmos con exponente menor a 1 que refleja tasas decrecientes a mayor porte, similar a los modelos de Shopping Centers del ITE.

También se estimaron tasas medias de generación de hipermercados en horas pico de viernes (Calle Adyacente) y sábado (Generador) para la tres variables independientes, siendo posible comparar las tasas expresadas por $\mathrm{m}^{2}$ de Área Total Construida con las tasas del uso del suelo 813 del ITE , según se muestra en la tabla 15 (Herz y Galarraga, 2013)

Tabla 15 - Tasas Medias (T.M.), Rango de tasas y Desviación Estándar (D.E.)

\begin{tabular}{c|c|c|c|c|c|c}
\hline \multirow{2}{*}{ ATC $(\mathrm{m} 2)$} & \multicolumn{3}{|c|}{ Tasa hora pico calle adyacente en } & \multicolumn{2}{c}{ Tasa hora pico Generador en Sábado } \\
& \multicolumn{3}{|c|}{ Viernes } & D. E. & Rango \\
\cline { 2 - 7 } & T.M. & D.E. & Rango & T.M. & D. & $0,0217-$ \\
Argentina & 0,0435 & 0,0150 & 0,0640 & 0,0225 & $0,0316-$ \\
(Galarraga et al.) & & & 0,0609 & & & 0,0913 \\
\hline EEUU & 0,0474 & 0,0255 & $0,0221-$ & 0,0608 & 0,0281 & $0,0322-$ \\
(ITE,2012) & & & 0,0798 & & & 0,0858 \\
\hline
\end{tabular}

\subsection{Distribución de viajes vehiculares atraídos y producidos durante días viernes y sábado}

Sobre la base de nuevos estudios de generación de viajes realizados en el marco de la Red Iberoamericana de Estudio de Polos Generadores de Viajes en hipermercados de Córdoba se obtuvieron datos de distribución horaria en días viernes y sábados. Como promedio de dos estudios de conteos en Hipermercados de Córdoba, la tabla 16 muestra los porcentajes de 
vehículos ingresando y saliendo de los hipermercados durante el día. El horario de 19 a 20 hs del viernes corresponde a la hora pico del tránsito adyacente, y el horarios entre las 20 y $21 \mathrm{hs}$ corresponde a la hora pico del Polo en día sábado.

Tabla 16 - Distribución horaria

\begin{tabular}{c|c|c|c|c|c}
\hline Hora & Viernes & Sábado & Hora & Viernes & Sábado \\
\hline$<9$ & $2,0 \%$ & $1,0 \%$ & $15-16$ & $5,8 \%$ & $5,5 \%$ \\
\hline $9-10$ & $2,9 \%$ & $1,9 \%$ & $16-17$ & $6,7 \%$ & $7,5 \%$ \\
\hline $10-11$ & $4,1 \%$ & $3,4 \%$ & $17-18$ & $8,0 \%$ & $9,1 \%$ \\
\hline $11-12$ & $4,8 \%$ & $4,4 \%$ & $18-19$ & $9,6 \%$ & $11,4 \%$ \\
\hline $12-13$ & $5,5 \%$ & $5,5 \%$ & $19-20$ & $12,0 \%$ & $13,3 \%$ \\
\hline $13-14$ & $5,0 \%$ & $5,8 \%$ & $20-21$ & $14,4 \%$ & $13,8 \%$ \\
\hline $14-15$ & $5,8 \%$ & $5,1 \%$ & $>21$ & $13,5 \%$ & $12,3 \%$ \\
\hline
\end{tabular}

\subsection{Prueba de modelo y tasa para hora pico del viernes}

Con objeto de evaluar la capacidad predictiva del modelo y tasa de hora pico del viernes con variable independiente ATC $\left(\mathrm{m}^{2}\right)$ de las Tablas 14 y 15, se efectuaron conteos y encuestas en un Hipermercado (H) inaugurado en el año 2010 con Área Total Construida de 11700 m2, ubicado cerca del límite de la cuidad. Los relevamientos se efectuaron de 18 a 20 hs en día viernes La Tabla 17 muestra la distribución modal del Hipermercado H comparada con la distribución media de anteriores estudios reportados en Tabla 12. El porcentaje de viajes vehiculares (autos + taxis) resultó 83,5\%, algo mayor al promedio de 75,7\%

Tabla 17 - Distribución modal

\begin{tabular}{c|c|c|c|c|c}
\hline Zona & $\begin{array}{c}\text { Auto } \\
(\%)\end{array}$ & $\begin{array}{c}\text { Taxi } \\
(\%)\end{array}$ & $\begin{array}{c}\text { Ómnibus } \\
(\%)\end{array}$ & A pie $(\%)$ & Otros $(\%)$ \\
\hline Híper H & 82,4 & 1,1 & $2 ., 9$ & 6,5 & 7,5 \\
\hline $\begin{array}{c}\text { Promedio } \\
\text { Tabla 12 }\end{array}$ & 69,6 & 6,2 & 5,5 & 13,7 & 4,9 \\
\hline
\end{tabular}

La Tabla 18 muestra la cantidad de vehículos contabilizados y la comparación con las estimaciones que producen el modelo y la tasa media correspondiente, para el porte del Polo y la hora pico del viernes en calles adyacentes (19 a 20hs). 
Tabla 18 - Cantidad de viajes en horas pico Viernes

\begin{tabular}{c|c|c|c|c|c}
\hline Hora & $\begin{array}{c}\text { Vehículos } \\
\text { entran }\end{array}$ & $\begin{array}{c}\text { Vehículos } \\
\text { salen }\end{array}$ & Total & $\begin{array}{c}\text { Estimados con } \\
\text { modelo de tabla 14 }\end{array}$ & $\begin{array}{c}\text { Estimados con tasa } \\
\text { de tabla 15 }\end{array}$ \\
\hline $18-19$ & 252 & 223 & 475 & - & - \\
\hline $19-20$ & 302 & 253 & 555 & 477 & 509 \\
\hline \multicolumn{7}{c}{ Diferencias } \\
\hline
\end{tabular}

Las diferencias encontradas son aceptables, y el hecho que el modelo y la tasa hayan subestimado los viajes vehiculares puede atribuirse a la mayor participación de viajes vehiculares registrada en este Polo respecto al promedio. En resumen cabe interpretar que los resultados del relevamiento del Hipermercado H, con ATC de 10700 m2, contribuyen a validar la tasa y modelo obtenidos localmente de día viernes en hora pico, de Tablas 14 y 15.

\subsection{Posibilidad de ajustar los modelos generalizados ITE para hipermercados de Córdoba}

Los modelos de generación de viajes de hipermercados en horas pico de viernes y sábado con variable ATC $\left(\mathrm{m}^{2}\right)$ fueron obtenidos sobre la base de 7 estudios, y reflejan una función de potencia linealizada con logaritmos con exponente menor a 1, similar a los modelos de Shopping Centers del ITE. Por otra parte, en la Tabla 15 se ha mostrado la similitud de tasas medias observadas en horas pico para hipermercados de Córdoba (Argentina) y para usos del suelo 813 ITE.

En este contexto cabe explorar la idea de aprovechar la sustancialmente mayor base de estudios del ITE, la convergencia de los modelos en el exponente $2 / 3$ y la propiedad de invariancia de escala de las funciones de potencia, para mejorar la modelización local de la generación de viajes de hipermercados.

La calibración de modelos extranjeros es un enfoque que presenta ventajas cuando permite aprovechar resultados experimentales de gran escala difíciles de reproducir localmente, y siempre que la dinámica general del fenómeno sea similar. La calibración no puede disminuir la dispersión pero si los sesgos. En este caso, se considera posible ajustar los parámetros $\alpha$ de la ecuación (5) a las características locales, para disponer de un modelo más general, potencialmente aplicable a otras dimensiones temporales de hipermercados y a otros usos del suelo comerciales. 
En la Tabla 19 se comparan los viajes estimados para un hipermercado de Córdoba de porte medio aplicando las tasas medias obtenidas en los estudios locales para horas pico de viernes (calle adyacente) y sábado (generador), y los viajes medidos en el Hipermercado $\mathrm{H}$, con los viajes estimados aplicando los modelos generalizados de la Tabla 9 obtenidos del ITE en las dimensiones temporales correspondientes. A efectos de la comparación, las superficies de Área Total Construida (GFA) se expresan en 1000 pies cuadrados $\left(\mathrm{Kp}^{2}\right)$.

Tabla 19 - Comparación de viajes estimados por distintos métodos

\begin{tabular}{c|c|c|c}
\hline Hipermercados en Córdoba & $\begin{array}{c}\text { Viernes, hora pico } \\
\text { en calle adyacente }\end{array}$ & $\begin{array}{c}\text { Sábado, hora pico } \\
\text { del generador }\end{array}$ & $\begin{array}{c}\text { Viernes Híper H, } \\
\text { hora pico en } \\
\text { calle adyacente }\end{array}$ \\
\hline Cantidad de estudios C.E. & 7 & 7 & 1 \\
\hline Rango de porte $\left(\mathrm{Kp}^{2}\right)$ & $60-250$ & $60-250$ & - \\
\hline GFA medio $\left(\mathrm{Kp}^{2}\right)$ & 178 & 178 & 126 \\
\hline $\begin{array}{c}\text { T.M. } \\
\text { (viajes generados/Kp }{ }^{2} \text { de GFA) }\end{array}$ & 4.05 & 5.95 & $555($ medidos $)$ \\
\hline $\begin{array}{c}\text { Viajes estimados con T.M. } \\
\text { local }\end{array}$ & 720 & 1059 & 704 \\
\hline $\begin{array}{c}\text { Viajes estimados con ecuación } \\
(5), \alpha \text { viernes: } 28, \alpha \text { sábado: } 40\end{array}$ & 886 & 1266 & $+27 \%$ \\
\hline Diferencia & $+23 \%$ & $+19 \%$ & \\
\hline
\end{tabular}

Se concluye que los modelos generalizados obtenidos del ITE pueden utilizarse localmente para horas pico de viernes y sábado de hipermercados de Córdoba, con el exponente 2/3 para la variable independiente.

Para calcular el correspondiente $\alpha$, debe tenerse en cuenta el cambio de unidades para X de $\mathrm{m}^{2}$ en lugar de $\mathrm{Kp}^{2}$ y las diferencias entre las estimaciones con los modelos generalizados y con la tasa media local de la Tabla 18.

Las ecuaciones (6) y (7) muestran entonces los nuevos modelos propuestos para generación de viajes de hipermercados en horas pico en Argentina

$\begin{array}{ll}\text { Viernes Hora Pico en Calle Adyacente } & \mathrm{Y}=1,11 \mathrm{X}^{2 / 3} \\ \text { Sábado Hora Pico de Generador } & \mathrm{Y}=1,59 \mathrm{X}^{2 / 3}\end{array}$

Dónde $\mathrm{X}=\mathrm{ATC}\left(\mathrm{m}^{2}\right)$ 
Lo interesante de estos modelos es su potencialidad de ser aplicables con los mismos parámetros para predecir generación de viajes en horas pico de viernes y sábados en supermercados y en Shopping Centers de Argentina, en la hipótesis que las dinámicas de estos polos comerciales son similares entre sí como se comprobó en Gran Bretaña y en el análisis de los usos del suelo 820, 850, 854 y 813 del ITE. La única diferencia es que para Shopping Centers la variable X corresponde al Área Total Rentable $\left(\mathrm{m}^{2}\right)$

En la hipótesis que la generación de viajes en día completo (de semana y sábado) también mantenga la relación del exponente $2 / 3$ a nivel local, queda abierta la posibilidad de obtener los parámetros $\alpha$ y validar los modelos correspondientes para hipermercados, supermercados y Shopping Centers en casos de Argentina.

\section{Conclusión}

El análisis de las ediciones 1991, 2008 y 2012 del Trip Generation Manual para los Usos del Suelo 850, 854 y 813 muestra que el Área Total Construida (Gross Floor Area, GFA) en miles de pies cuadrados $\left(\mathrm{Kp}^{2}\right)$ es la variable independiente seleccionada para explicar la generación de viajes. Se advierte que las Tasas Medias por GFA son mayores para el Uso del Suelo 850, algo menores para el suelo 854 y mucho menores para el Uso de Suelo 813. Es decir, las tasas disminuyen a medida que aumenta el área total construida. Las tasas de la edición ITE 2012 son menores a las correspondientes al ITE 2008. Los porcentajes de reducción son variables, pero en términos generales resultan del 5 al 30\% para el Uso del Suelo 850, del 5 al 15\% para el Uso del Suelo 854 y del 5 al 10\% para el Uso del Suelo 813.

Para cuatro dimensiones temporales, se analizaron los estudios reportados de generación viajes de Shopping Centers del ITE (uso del suelo 820) que abarcan rangos de superficies muy amplios de 100 a $2200 \mathrm{Kp} 2$ y son mucho más numerosos que los estudios reportados para los supermercados e hipermercados. Los modelos de generación de viajes son linealizaciones logaritmicas de funciones de potencia con exponentes entre 0,63 y 0,67 , que reflejan disminución de tasa de generación con el aumento de porte del Polo, similar a lo observado en supermercados e hipermercados. Pudo verificarse que estos modelos reproducen razonablemente las tasas medias tanto de supermercados, supermercados de cadenas regionales e hipermercados, para el porte medio representativo de cada uso del suelo. Vista la 
similitud de exponentes para las cuatro dimensiones temporales, se ha propuesto el modelo generalizado referido en la ecuación (5).

La constancia del exponente 2/3 aplicado al Área Total Construida como ley de crecimiento de viajes vehiculares implica que la concentración de la oferta comercial genera menos cantidad de viajes vehiculares por compras en la ciudad, aunque podrían ser de mayor distancia.

Se ha verificado la aplicabilidad de los modelos generalizados a estudios de hipermercados realizados en Argentina. Para su formulación se estimó el parámetro $\alpha$, teniendo en cuenta el cambio de unidades para $\mathrm{X}$ de $\mathrm{m}^{2}$ en lugar de $\mathrm{Kp}^{2}$, y las diferencias de las predicciones modeladas con la tasa media local. Los modelos generalizados propuestos para hipermercados de Argentina son los referidos en las ecuaciones (6) y (7).

Con pruebas de validación adecuadas, estos modelos tienen potencialidad de ser aplicables para estimar la generación de viajes de supermercados y Shopping Centers de Argentina, en diferentes dimensiones temporales, con el mismo exponente y calibrando el parámetro $\alpha$. La diferencia es que para Shopping Centers la variable X es el Área Total Rentable $\left(\mathrm{m}^{2}\right)$ y para supermercados e hipermercados es el Área Total Construida $\left(\mathrm{m}^{2}\right)$. Se considera que, aún perdiendo especificidad para cada uso del suelo en particular, la modelación propuesta gana en generalidad y aplicabilidad con base de datos reducidos, como es el caso en Argentina.

\section{Referencias}

Baker, A. (2004) Simplicity. Stanford Encyclopedia of Philosophy. Available at plato.stanford.edu.

Brehmer, C. L. y Butorac, M. A. (2003) Trip Generation Characteristics of Discount Supermarkets. ITE Journal, vol. 73, n. 11.

Freitas G. V. y Raia Jr.A.A (2008) Metodologia para Avaliação de Impactos na Implantação de Supermercados em Cidades Medias. XXII ANPET, Fortaleza, Brasil

Galarraga, J., Herz, M., Raia, A. y Freitas, G. (2011) Caderno Pólos Geradores de Viagens Orientados à Qualidade de Vida e Ambiental: Hipermercados, http://redpgv.coppe.ufrj.br

Galarraga, J., Herz, M., Albrieu, L., Depiante, V. y Pastor, G. (2007) Características de los Viajes en Hipermercados de la Ciudad de Córdoba - Argentina. XIV Congreso Latinoamericano de Transporte Público y Urbano CLATPU, Rio de Janeiro, Brasil

Herz, M. y Galarraga, J. (2013) Findings on Trip Generation Hubs in Argentina. The 13th World Conference on Transport Research WCTR, Rio de Janeiro, Brasil 
Institute of Transportation Engineers - ITE (1991). Trip Generation, 5th Edition, Washington, D.C., U.S.A.

Institute of Transportation Engineers - ITE (2008) Trip Generation, 8th Edition, Washington, D.C., U.S.A.

Institute of Transportation Engineers - ITE (2012). Trip Generation, 9th Edition, Washington, D.C., U.S.A.

Milne, A., Abley, S. y Douglass, M. (2009). Comparison of NZ and UK trips and parking rates. Land Transport NZ Research Report 374, Wellington, Nueva Zelandia

Pearson, D. F., Bochner, B. S., Ellis, P. y Ojah, M. (2009) Discount Superstore Trip Generation . ITE Journal vol.79 n $\%$

Portugal, L. S. (2012) Polos Geradores de Viagens Orientados a Qualidade de Vida e Ambiental: Modelos y taxas de geração de viagens. Ed. Interciencia, Rio de Janeiro, Brasil

Silva, L. R., Silva P. C. (2006) Modelos de Geração de Viagens Endoexógenos para Polos Generadores de Viagens-Estudo de caso nos Supermercados e Hipermercados. XX ANPET, Brasília, Brasil 